\title{
A SEMIMODULAR IMBEDDING OF LATTICES
}

\author{
D. T. FINKBEINER
}

1. Introduction. The study of structural or arithmetic properties of a general lattice $\&$ often can be facilitated by imbedding $\&$ as a sublattice of a lattice $\subseteq$ of a more restricted type whose properties are known. However, if $\widetilde{\Xi}$ is too restricted, a general imbedding is impossible; for example, $\widetilde{\Xi}$ cannot be modular because $\mathbb{R}$, as a sublattice of $\subseteq$, would then have to be modular. One of the best results of this nature has been given by Dilworth in an unpublished work in which he shows that any finite dimensional lattice is isomorphic to a sublattice of a semi-modular point lattice (1, pp. 105 and 110 ). In the present paper Dilworth's imbedding process is modified to obtain a sharper result: Any finite dimensional lattice $?$ is isometrically isomorphic to a sublattice of a semi-modular lattice $\subseteq$ which has the same number of points as $\mathbb{R}$ and which preserves basic properties of the join-irreducible arithmetic of $\mathbb{R}$.

Although the meet-irreducible arithmetic of semi-modular lattices is known (2), a corresponding theory of join-irreducible arithmetic remains to be developed. The work of this paper suggests that a knowledge of the joinirreducible arithmetic of semi-modular lattices would provide a corresponding theory for all finite dimensional lattices.

Aside from possible applications to lattice arithmetic, the imbedding process is of intrinsic interest. First a pseudo-rank function $s$ is defined on $?$. Then $(\S 3) \mathbb{R}$ is imbedded as a sublattice of a lattice $\mathfrak{M}$ which is constructed from $\mathbb{R}$ by introducing between each join irreducible $q \in \Omega$ and the element $c$ which is covered by $q$ a chain of $s(q)-s(c)-1$ elements which are both join and meet irreducible in $\mathfrak{M}$. The function $s$ is extended to $\mathfrak{M}$. In $\S \S 4$ and 5 normal subsets of the set $Q$ of all join irreducibles of $\mathfrak{M}$ are used to define a dependence relation on $Q$. Finally ( $\S 6)$ the subsets of $Q$ which are closed relative to this dependence relation form a semi-modular lattice $\subseteq$ whose join irreducibles are order-isomorphic to $Q$; $\subseteq$ contains a sublattice which is isomorphic to $?$, and

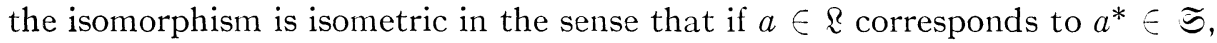
then $s(a)$ is the ordinary rank of $a^{*}$ in $\subseteq$.

2. Pseudo-rank functions. If $\subseteq$ is a semi-modular lattice of finite dimension, then the usual rank function $r$ on $\subseteq$ has the properties

$$
\begin{aligned}
& r(z)=0, \\
& \text { if } a \text { covers } b(a>b) \text {, then } r(a)=r(b)+1,
\end{aligned}
$$

Received May 20, 1959. Final composition of this paper was performed while the author was a National Science Foundation Faculty Fellow at Princeton University. The author gratefully acknowledges the influence of R. P. Dilworth upon the results obtained here. 


$$
r(a)+r(b) \geqslant r(a \cup b)+r(a \cap b) .
$$

Furthermore, if $\mathbb{R}$ is any sublattice of $\subseteq$, then on $\&$ the function $r$ satisfies (2.3) and

$$
a \supset b \text { implies } r(a)>r(b) \text {. }
$$

THEOREM 2.1. If $\mathrm{R}$ is any finite dimensional lattice, there exists an integral valued function defined on $\&$ which satisfies (2.1), $\left(2.2^{*}\right)$, and (2.3).

Proof. Let $u$ and $z$ denote the unit and null elements of $\&$. For $a \in \mathbb{R}$ let $m(a)$ be the maximal length of all chains from $a$ to $z$, and let $s(a)=2^{m(u)}$ $-2^{m(u)-m(a)}$. It is readily verified that $s$ satisfies the conditions stated.

Any function which satisfies the conditions of Theorem 2.1 will be called a pseudo-rank function.

3. Extension of $\mathfrak{R}$. Let $\mathfrak{R}$ be any finite dimensional lattice and $s$ any pseudo-rank function on $\mathbb{R}$. The next objective is to imbed $\mathbb{R}$ as a sublattice in a lattice $\mathfrak{M}$ which has more join irreducibles but otherwise retains the arithmetic properties of $\mathbb{R}$. Each irreducible $q \in \mathbb{Z}$ covers a uniquely determined element $c$. Let $k=s(q)-s(c)-1$. Whenever $k>0$ introduce between $q$ and $c$ a construction chain of $k$ new elements $q_{i}$,

$$
q>q_{1}>q_{2}>\ldots>q_{k}>c .
$$

Only the maximal and minimal elements of each such chain belong to $\mathbb{R}$, and distinct chains are either disjoint or have only the minimal element in common.

Let the set $\mathfrak{M}$ consist of the elements of $\mathbb{\&}$ together with the non-extremal elements of the construction chains. It is easy to define formally the ordering described above for $\mathfrak{M}$ by superimposing the ordering of $\mathbb{R}$ and that of the construction chains. Then $\mathfrak{M}$ is a lattice in which the non-extremal elements of the construction chains are both meet and join irreducible, and in which the remaining elements form a sublattice isomorphic to $\mathfrak{\Omega}$. The join irreducible elements of $\mathfrak{M}$ are those of $\mathfrak{Z}$ together with all non-extremal elements of the construction chains; thus $\mathfrak{M}$ and $\mathbb{R}$ have the same number of points.

The function $s$ on $\mathbb{R}$ is extended to a function $r$ on $\mathfrak{M}$ by defining

$$
\begin{aligned}
r(b) & =s(b) \quad \text { if } b \in \mathfrak{R} \\
& =s\left(b_{2}\right)+j \quad \text { if } b \notin \mathbb{R},
\end{aligned}
$$

where $b_{2}$ is the minimal element of the construction chain in which $b$ appears, and where $j$ is the length of that chain from $b$ to $b_{2}$. Clearly $r$ satisfies (2.1) and $\left(2.2^{*}\right)$; furthermore, $(2.3)$ is satisfied by every $a$ which is join irreducible in $\mathfrak{M}$.

4. Normal sets of irreducibles. Let $Q$ denote the set of all join irreducible elements $q \neq z$ of $\mathfrak{M}$. For any $S \subseteq Q$ let $n(S)$ denote the number of elements 
in $S$, and for each $b \in \mathfrak{M}$ let $Q_{b}=\{q \in Q \mid q \subseteq b\}$. Clearly $Q_{a} \wedge Q_{b}=Q_{a n b}$ but the corresponding equality for union is not valid.

Definition 4.1. A subset $S \subseteq Q$ is said to be normal if and only if the following two conditions are satisfied:

$$
\begin{array}{ll}
\left(\mathrm{N}_{1}\right) & \text { If } R \subset S \text {, then } n(R) \leqslant r(\cup R), \\
\left(\mathrm{N}_{2}\right) & n(S)=r(\cup S) .
\end{array}
$$

Normal sets are determined not only by the structure of $\mathfrak{M}$ but also by the function $r$ which is not uniquely determined by $\mathfrak{M}$. The following lemma provides the fundamental tool for later proofs.

LEMmA 4.1. If $S$ and T are normal sets such that $s=\cup S \in \mathbb{R}$ and $t=\cup T \in \mathbb{R}$, there exists a normal set $N \subseteq S \vee T$ such that $\cup N=s \cup t$.

Proof. Since $\cup(S \wedge T) \subseteq s \cap t$, we have

$$
\begin{aligned}
n(S \vee T) & =n(S)+n(T)-n(S \wedge T) \\
& \geqslant r(s)+r(t)-r(\cup(S \wedge T)) \\
& \geqslant r(s)+r(t)-r(s \cap t) \geqslant r(s \cup t)
\end{aligned}
$$

An inductive argument is used to show that $S \vee T$ contains a normal subset $N$ such that $\cup N=s \cup t$. Let $b \in \mathfrak{M}$ be minimal such that $s \subset b \subseteq s \cup t$ and $r(b)-r(s) \leqslant n\left(T \wedge\left(Q_{b}-Q_{s}\right)\right)$. Since $s \cup t$ satisfies these two requirements, such minimal elements exist. Choose $B \subseteq T \wedge\left(Q_{b}-Q_{s}\right)$ such that $n(B)=r(b)-r(s)$, and let $R=S \vee B$. Then $S$ and $B$ are disjoint, $\cup R \subseteq b$, and $n(R)=r(b)$. To prove that $R$ is normal, it suffices to show that $W \subseteq R$ implies $n(W) \leqslant r(\cup W)$, for then $n(R) \leqslant r(\cup R) \leqslant r(b)=n(R)$.

Suppose that $W \subseteq R$ exists such that $n(W)>r(\cup W)$. Write $W=S^{\prime} \vee B^{\prime}$, where $S^{\prime} \subseteq S$ and $B^{\prime} \subseteq B$. Clearly $S^{\prime}$ and $B^{\prime}$ are disjoint, and $B^{\prime}$ may be assumed to be non-void since otherwise $W$ is a subset of the normal set $S$. Also $w=\cup W \subseteq s$, since $B^{\prime}$ is non-void and disjoint from $Q_{s}$. First suppose $w \notin R$; that is, $w$ is an irreducible of $\mathfrak{M}$ introduced by the construction chains. Let $w_{1} \in \mathbb{R}$ be the minimal element of the construction chain $C$ in which $w$ appears. Then

$$
B^{\prime}=\left(B^{\prime} \wedge Q_{w_{1}}\right) \vee\left(B^{\prime} \wedge C\right),
$$

is a disjoint union. Clearly $n\left(B^{\prime} \wedge C\right) \leqslant r(w)-r\left(w_{1}\right)$. Thus

$$
\begin{aligned}
& r(w)<n(w)=n\left(S^{\prime}\right)+n\left(B^{\prime}\right) \leqslant n\left(S^{\prime}\right)+n\left(B^{\prime} \wedge Q_{w_{1}}\right)+r(w)-r\left(w_{1}\right), \\
& r\left(w_{1}\right)<n\left(S^{\prime}\right)+n\left(B^{\prime} \wedge Q_{w_{1}}\right) .
\end{aligned}
$$

Let $W^{\prime}=S^{\prime} \vee\left(B^{\prime} \wedge Q_{w 1}\right)$, and let $w^{\prime}=\cup W^{\prime}$. Since $\cup S^{\prime} \subseteq w_{1}$, we have $w^{\prime} \subseteq w_{1}$. Then $n\left(W^{\prime}\right)>r\left(w^{\prime}\right)$. If $w^{\prime} \notin \Omega$, the argument may be repeated, reducing $W^{\prime}$ to a smaller set. This process must end before all the elements of $B^{\prime}$ are removed because otherwise $n\left(S^{\prime}\right)>r\left(\cup S^{\prime}\right)$, contradicting the fact 
that $S^{\prime}$ is a subset of the normal set $S$. Hence we need to consider only the case for which $W \subseteq R, n(W)>r(\cup W)$, and $\cup W \in$ \&. Then

$$
\begin{aligned}
\cup S^{\prime} & =\cup(W \wedge S) \subseteq(\cup W) \cap(\cup S)=w \cap s, \\
r(w)<n(W) & =n\left(S^{\prime}\right)+n\left(B^{\prime}\right) \leqslant r\left(\cup S^{\prime}\right)+n\left(B^{\prime}\right) \\
& \leqslant r(w \cap s)+n\left(B^{\prime}\right) \leqslant r(w)+r(s)-r(w \cup s)+n\left(B^{\prime}\right) \\
& =r(w)+n(S)-r(w \cup s)+n\left(B^{\prime}\right) .
\end{aligned}
$$

Hence $r(w \cup s)<n(S)+n\left(B^{\prime}\right) \leqslant n(S)+n(B)=n(R)=r(b)$. Since $w \subseteq s$, we have $s \subset w \cup s \subset b$. Also $B^{\prime} \subseteq T \wedge\left(Q_{w \cup_{s}}-Q_{s}\right)$, and therefore

$$
r(w \cup s)-r(s)<n\left(B^{\prime}\right) \leqslant n\left(T \wedge\left(Q_{w \cup s}-Q_{s}\right)\right) .
$$

But this contradicts the minimal property assumed for $b$. Thus the normal set $S$ has been extended to a normal set $R$ by adjoining certain elements of the normal set $T$. The argument can be iterated, replacing $S$ by $R$, to construct a normal set $N \subseteq S \vee T$ such that $\cup N=s \cup t$.

Observe that in this proof $S$ was augmented by elements of $T$ to produce a normal set $N=S \vee T^{\prime}$, where $T^{\prime} \subseteq T$. The roles of $S$ and $T$ could have been interchanged, so there also exists a normal set $N^{\prime}=S^{\prime} \vee T$, where $S^{\prime} \subseteq S$ and $\cup N^{\prime}=\cup N=s \cup t$.

Lemma 4.2. For each $b \in \mathfrak{M}$ there exists a normal set $B$ such that $\cup B=b$.

Proof. This is trivial for all points of $\mathfrak{M}$; we proceed by induction. Let $r(b)=k$, and assume that the lemma holds for all $a \in \mathfrak{M}$ for which $r(a)<k$. If $b$ is irreducible, $b>c$, and by the induction hypothesis there exists a normal set $C$ for which $c=\cup C$. Then $B=C \vee(b)$ is normal and $b=\cup B$. If $b$ is reducible, then $b \in \Omega$ and $b=s \cup t$ for suitable $s, t \in \Omega$. By the induction hypothesis there exist normal sets $S$, T with $s=\cup S$ and $t=\cup T$, and Lemma 4.1 then guarantees the existence of a normal set $B \subseteq S \vee T$ such that $\cup B=b$.

5. The normal dependence relation. The next step in the imbedding process is to define a dependence relation $\Delta$ between the elements and subsets of $Q$ and to develop its properties. For $S \subseteq Q$, let

$$
S^{*}=\left\{q^{*} \in Q \mid q^{*} \subseteq q \text { for some } q \in S\right\} .
$$

Definition 5.1. An irreducible $q \subseteq Q$ is said to depend normally on a subset $S \subseteq Q$, written $q \Delta S$, if and only if $q \subseteq \cup T$ for some normal set $T \subseteq S^{*}$. (The notation $P \Delta R$ is used to mean $q \Delta R$ for every $q \in P$.)

As immediate consequences of this definition we have

$$
\begin{aligned}
& S^{*} \Delta S \text { for every non-void } S \subseteq Q, \\
& \text { if } S \Delta T \text {, then } S^{*} \Delta T, \\
& \text { if } q \Delta S \text {, then } q \subseteq \cup S .
\end{aligned}
$$


LEMma 5.1. $\Delta$ also satisfies

$$
q^{\prime} \subseteq q \text { implies } q^{\prime} \Delta S \vee q \text { for any } S \subseteq Q,
$$$$
q \Delta S \text { and } S \Delta T \text { imply } q \Delta T \text {, }
$$

$q^{\prime} \Delta q$ implies $q^{\prime} \subseteq q$,

$q \Delta S$ and $S \Delta q$ imply $q \in S$,

if $q^{\prime \prime} \subset q^{\prime}$ implies $q^{\prime \prime} \Delta S$, then $q \Delta S \vee q^{\prime}$ implies either $q \Delta S$ or $q^{\prime} \Delta S \vee q$.

Proof. $(\Delta 1)$ follows directly from (5.1), while $(\Delta 3)$ and $(\Delta 4)$ both follow from (5.3). Consider $(\Delta 2)$, and let $q \Delta S$ and $S \Delta T$; there exists a normal set $M \subseteq S^{*}$ such that $q \subseteq \cup M=m$. By (5.2) $M \Delta T$. If $m \notin R, m$ must be join-irreducible, which implies $q \in S^{*}$ and $q \Delta T$. Hence consider $m \in \mathcal{E}$. Write $M$ as a disjoint union, $M=\left(M \wedge T^{*}\right) \vee M_{1}$. If $M_{1}$ is void, $M \subseteq T^{*}$, and $q \Delta T$. Otherwise for each $q_{i} \in M_{1}$ there exists a normal set $T_{i} \subseteq T^{*}$ such that $q_{i} \subseteq \cup T_{i}=t_{i}$. If $t_{i} \notin \Omega$ for some $q_{i} \in M_{1}$ then $t_{i}$ is join-irreducible, and $q_{i} \in T^{*}$, contrary to $q_{i} \in M_{1}$. Hence $t_{i} \in \&$ for each $i$. Apply Lemma 4.1 a finite number of times to obtain a normal set

$$
R \subseteq \underset{q i \in M_{1}}{\vee} T_{i}
$$

such that $\cup R=\cup t_{i}=t \in R$. Clearly $R \subseteq T^{*}$. Apply Lemma 4.1 again to $R$ and $M$ to obtain a normal set $N$ of the form $N=R \vee M^{\prime}$, where $M^{\prime} \subseteq M$ and $\cup X^{*}=t \cup m$. Then $N \subseteq T^{*}$, for if $q_{i} \in N^{*} \wedge M_{1}$, then

$$
\cup\left(R \vee q_{i}\right)=t \cup q_{i} \subseteq t \cup t_{i}=t .
$$

Hence

$$
r\left(\cup\left(R \vee q_{i}\right)\right)=r(t)<r(t)+1=n(R)+1=n\left(R \vee q_{i}\right),
$$

which contradicts the normality of $N$. But $q \subseteq m \subseteq \cup N$, where $N$ is a normal subset of $T^{*}$, so $q \Delta T$.

To verify $(\Delta 5)$, assume that $q^{\prime \prime} \Delta S$ for all $q^{\prime \prime} \subset q^{\prime}$, that $q \Delta S \vee q^{\prime}$, but that $q \Delta S$. Then by $(5.1)$ and $(\Delta 2) q^{\prime}$ is the only element of $\left(S \vee q^{\prime}\right)^{*}$ which does not depend normally on $S$. By the definition of $\Delta$, there exists a normal set $T \subseteq\left(S \vee q^{\prime}\right)^{*}$ such that $q \subseteq \cup T=t$. Since $q \Delta T$, we may assume $q^{\prime} \in T$, for otherwise $T \Delta S$, from which follows $q \Delta S$, contrary to hypothesis. Thus we write $T=T^{\prime} \vee q^{\prime}$, where $T^{\prime} \Delta S$. We assert that $T^{\prime} \vee q$ is normal and $t=\cup\left(T^{\prime} \vee q\right)$. Let $R$ be any subset of $T^{\prime} \vee q$. If $R \subseteq T^{\prime}$, then $R \subseteq T$, so $n(R) \leqslant r(\cup R)$ since $T$ is normal. If $R \not \subset T^{\prime}$, then $R=R^{\prime} \vee q$, where $R^{\prime} \subseteq T^{\prime} \subseteq T$. Then

$$
n\left(R^{\prime}\right)=n(R)-1 \leqslant r\left(\cup R^{\prime}\right) \leqslant r(\cup R) .
$$

Suppose $R_{0}=R_{0}^{\prime} \vee q \subseteq T^{\prime} \vee q$ exists such that $n\left(R_{0}\right)-1=r\left(\cup R_{0}\right)$. Then from (5.4), $n\left(R_{0}{ }^{\prime}\right)=n\left(R_{0}\right)-1=r\left(\cup R_{0}{ }^{\prime}\right)=r\left(\cup R_{0}\right)$. Since 
$R_{0}{ }^{\prime} \subseteq T^{\prime} \subseteq T, R_{0}{ }^{\prime}$ is normal. Also $q \subseteq \cup R_{0}=\cup R_{0}{ }^{\prime}$. Hence $q \Delta R_{0}{ }^{\prime}$; but $R_{0}{ }^{\prime} \Delta S$, so $q \Delta S$ which is a contradiction. Thus from (5.4) we obtain $n(R) \leqslant r(\cup R)$ for all $R \subseteq T^{\prime} \vee q$, and $T^{\prime} \vee q$ satisfies $\left(\mathrm{N}_{1}\right)$. But since $T=T^{\prime} \vee q^{\prime}$ is normal and $q \subseteq \cup T$,

$$
\begin{aligned}
n\left(T^{\prime} \vee q\right)=n\left(T^{\prime}\right)+1 & \leqslant r\left(\cup\left(T^{\prime} \vee q\right)\right) \\
& \leqslant r(\cup T)=r\left(\cup\left(T^{\prime} \vee q^{\prime}\right)\right) \\
& =n(T)=n\left(T^{\prime}\right)+1 .
\end{aligned}
$$

Therefore, both of these inequalities must be equalities, and

$$
n\left(T^{\prime} \vee q\right)=r\left(\cup\left(T^{\prime} \vee q\right)\right)=r(\cup T) .
$$

Thus $T^{\prime} \vee q$ satisfies $\left(\mathrm{N}_{2}\right)$ and is normal. But also $\cup\left(T^{\prime} \vee q\right) \subseteq \cup T$, so (5.5) implies that equality holds. Since $q^{\prime} \in T, q^{\prime} \subseteq \cup\left(T^{\prime} \vee q\right)$. Hence $q^{\prime} \Delta T^{\prime} \vee q$, and $q^{\prime} \Delta S \vee q$. This completes the proof of Lemma 5.1.

6. The normal imbedding. It was shown by the author (3) that any relation $\Delta$ on a partially ordered set $Q$ determines a complete semi-modular lattice $\subseteq$ whose set of join irreducibles is order isomorphic to $Q$, provided $\Delta$ satisfies the five properties of Lemma 5.1. The elements of $\subseteq$ are the closed subsets of $Q$ where $S \subseteq Q$ is said to be closed if and only if $q \Delta S$ implies $q \in S$. The closed subsets determined by the normal dependence relation form the imbedding lattice which was described in the introduction. Recall that for each $b \in \mathfrak{M}, Q_{b}$ denotes the set of all $q \in Q$ such that $q \subseteq b$.

ThEOREM. Let $\subseteq$ be the lattice of all subsets of $Q$ which are closed under the normal dependence relation. Then

(6.1) $\subseteq$ is a complete semi-modular lattice whose set of join irreducibles is isomorhphic to $Q$ under the mapping $q \rightarrow Q_{\eta}$,

(6.2) $\subseteq$, M, and $\mathbb{Q}$ have the same number of points,

(6.3) the mapping $b \rightarrow Q_{b}$ is a one-to-one mapping of $\mathfrak{M}$ onto a lattice within $\subseteq$ and an isomorphism of $\mathbb{R}$ onto a sublattice of $\subseteq$,

(6.4) for every $b \in \mathfrak{M}, r(b)$ is the ordinary rank of $Q_{b}$ in $\subseteq$,

(6.5) properties of the join arithmetic of $\mathbb{Q}$ are preserved in $\subseteq$.

Proof. The precise meaning of $(6.5)$ is contained in the statement of Lemma 6.4. Theorem 3 of (3) establishes (6.1). Hence $\subseteq$ and $\mathfrak{M}$ (and therefore $\mathbb{R}$ ) have the same number of points. The remaining statements are established by a sequence of lemmas.

Lemma 6.1. For each $b \in \mathfrak{M}, Q_{b} \in \cong$.

Proof. Since $Q_{b}=Q_{b}{ }^{*}$ and $b=\cup Q_{b}, Q_{b}$ is closed for each $b \in \mathfrak{M}$.

Lemma 6.2. For all $a, b \in \mathfrak{R}, Q_{a} \cup Q_{b}=Q_{a \cup b}$.

Proof. $Q_{c} \cup Q_{b}$ is the smallest closed set containing $Q_{a} \vee Q_{b}$; hence $Q_{a} \cup Q_{b} \subseteq Q_{a \cup b}$. By Lemma 4.2, there exist normal sets $A \subseteq Q_{a}$ and $B \subseteq Q_{b}$ 
such that $\cup A=a$ and $\cup B=b$. Apply Lemma 4.1 to obtain a normal set $N \subseteq A \vee B$ such that $\cup N=\cup(A \vee B)=a \cup b$. If $q \in Q_{a \cup b}$ then $q \Delta N$. This implies $Q_{a \cup b} \subseteq Q_{a} \cup Q_{b}$, so equality holds.

Thus the mapping $b \rightarrow Q_{b}$ preserves joins of elements of $\&$; since it also preserves intersections, $\mathbb{R}$ is mapped isomorphically onto a sublattice of $\mathbb{S}$. Clearly, for $a, b \in \mathfrak{M}, a \subseteq b$ if and only if $Q_{a} \subseteq Q_{b}$. Hence the mapping of $\mathfrak{M}$ into $\subseteq$ is order-preserving. Intersections are preserved, but joins are not, in general. Since $Q_{a} \vee Q_{b} \subseteq Q_{a \cup b}$, the image of $\mathfrak{M}$ forms within $\subseteq$ a lattice which is isomorphic to $\mathfrak{M}$ but which is not necessarily a sublattice of $\Xi$.

To prove (6.4) we use an inductive argument. For $b \in \mathfrak{M}$ if $r(b)=1$, then $Q_{b}=(b)$ is a point of $\subseteq$. Suppose the rank in $\subseteq$ of $Q_{c}$ is $r(c)$ for all $c \subset b$. If $b$ is irreducible and $b>c$, then $Q_{b}=Q_{c} \vee b>Q_{c}$ in $\Subset$, so $r(b)=r(c)+1$ is the rank of $Q_{b}$ in $\subseteq$. If $b$ is reducible in $\mathfrak{M}$, then $b \in \mathfrak{R}$. Let $b>c$ in $\mathbb{R}$ and let $q \in \Omega$ be such that $q>c \cap q$ in $\&$. Then

$$
j=r(b)-r(c) \leqslant r(q)-r(c \cap q)=k .
$$

In $\mathfrak{M}$ there exists a chain $q=q_{k}>q_{k-1}>\ldots>q_{1}>c \cap q$. Let $S_{i}=Q_{c} \vee q_{1}$ $\vee \ldots \vee q_{i}$ for $1 \leqslant i \leqslant k$. Then we assert

(a) $S_{1}, S_{2}, \ldots, S_{j-1}$ are closed, and

(b) $Q_{b} \Delta S_{j}$.

If these two statements are valid, then in $\subseteq$ we have

$$
Q_{b}>S_{j-1}>\ldots>S_{1}>Q_{c},
$$

so the rank of $Q_{b}$ is $r(b)=r(c)+j$. Thus (6.4) follows from the next lemma.

LemMa 6.3. In \& let $b>c$, where $b$ is reducible, and let $q_{k} \subset b$ be such that $q_{k}>q_{k} \cap c$. Let the construction chain in $\mathfrak{M}$ which is headed by $q_{k}$ be $q_{k}>q_{k-1}$ $>\ldots>q_{1}>q_{k} \cap c$, where $k \geqslant r(b)-r(c)=j$. Let $S_{i}=Q_{c} \vee q_{1} \vee \ldots \vee q_{i}$ for $i \leqslant j$. Then

(a) $S_{1}, \ldots, S_{j-1}$ are closed, and

(b) $Q_{b} \Delta S_{j}$.

Proof. For $i<j$ let $q \Delta S_{i}$; there exists a normal subset $N \subseteq S_{i}{ }^{*}=S_{i}$ such that $q \subseteq \cup N$. First assume $\cup N \in \mathbb{R}$ and $c \cup \cup N=b$. Let $M \subseteq Q_{c}$ be normal such that $\cup M=c$. By Lemma 4.1 extend $V$ by adjoining elements of $M$ to obtain a normal set $B \subseteq S_{i}$ for which $\cup B=b$. Then

$$
n\left(B \wedge Q_{c}\right) \geqslant n(B)-i=r(b)-i>r(b)-j=r(c) \geqslant r\left(\cup\left(B \wedge Q_{c}\right)\right) \text {. }
$$

This contradicts the normality of $B$. Hence either $\cup N \notin R$ or $c \cup \cup M \subset b$. In the latter case $\cup N \subseteq c$ since $b>c$. Then $q \Delta N \subseteq Q_{c} \subseteq S_{i}$. If $c \cup \cup N=b$ then $\cup N \notin R$, so $\cup N=q_{s}$ for some $s=1, \ldots, i$. Then

$$
q \Delta N \subseteq Q_{q_{s}} \subseteq S_{i}
$$

Hence $S_{i}$ is closed. 
To prove (b) we show that $P=M \vee q_{1} \vee \ldots \vee q_{j}$ is normal. Since $\cup P=b$ it will follow that $q \Delta P \subseteq S_{j}$ for all $q \in Q_{b}$. Clearly

$$
n(P)=n(M)+j=r(c)+j=r(b) .
$$

Let $T \subseteq P$, and write $T$ as a disjoint union,

$$
T=C \vee D \text {, where } C \subseteq M \text { and } D \subseteq\left\{q_{1}, \ldots, q_{j}\right\} .
$$

If $\cup T \notin \Omega$, either $D$ is void or $\cup T=q_{s}$ for some $s=1, \ldots, j$. In the former case $T \subseteq M$ so $r(\cup T) \leqslant n(T)$; in the latter case

$$
T \subseteq Q_{q_{s}} \subseteq\left(q_{1} \vee \ldots \vee q_{s}\right) \vee\left(C \wedge Q_{c \cap q_{k}}\right)
$$

But

$$
n\left(C \wedge Q_{c \cap q_{k}}\right) \leqslant r\left(c \cap q_{k}\right)
$$

since $C \subseteq M$. Thus

$$
n(T) \leqslant s+r\left(c \cap q_{k}\right)=r\left(q_{s}\right)=r(\cup T) .
$$

Finally suppose $\cup T \in \mathfrak{R}$. Then

$$
n(T)=n(C)+n(D) \leqslant r(\cup C)+j \leqslant r(C \cap \cup T)+r(b)-r(c),
$$

since $\cup C \subseteq c \cap \cup T$. But since $r$ satisfies (2.2) on $\mathfrak{R}$,

$$
r(c \cap \cup T) \leqslant r(c)+r(\cup T)-r(c \cup \cup T) .
$$

Hence $n(T) \leqslant r(\cup T)$, and $P$ is normal. This completes the proof of Lemma 6.3 and consequently (6.4).

Lemma 6.4. For $b \in \Omega$ and $q_{i}^{*} \in Q$, let

$$
Q_{b}=\bigcup_{i=1}^{m} Q_{q_{i}}{ }^{*}
$$

be a reduced join representation having the least possible number of components. Then there exist $q_{i} \in \mathfrak{R}, i=1, \ldots, m$, such that both

$$
b=\bigcup_{i=1}^{m} q_{i}
$$

and

$$
Q_{b}=\bigcup_{i=1}^{m} Q_{q_{i}}
$$

are reduced representations.

Proof. From the isomorphism, for $q \in \Omega$ the representation

$$
b=\bigcup_{i=1}^{m} q_{i}
$$

is reduced in $\&$ if and only if

$$
Q_{b}=\bigcup_{i=1}^{m} Q_{q_{i}}
$$


is reduced in $\subseteq$. Thus the join representations in $\mathbb{R}$ are carried intact into $\subseteq$; however, some irreducibles of $\subseteq$ may not be the image of an irreducible of R. If

$$
Q_{b}=\bigcup_{i=1}^{m} Q_{q_{i}}{ }^{*}
$$

for $q_{i}{ }^{*} \in Q$, let $q_{i} \in \Omega$ be the maximal element of that construction chain in which $q_{i}{ }^{*}$ appears. Then

$$
Q_{b}=\bigcup_{i=1}^{m} Q_{q_{i}} .
$$

If no representation of $Q_{b}$ has fewer than $m$ components, this representation is reduced, as is

$$
b=\bigcup_{i=1}^{m} q_{i}
$$

This completes the proof of the main theorem.

Our concluding remarks are directed to the problem of determining in what sense the normal imbedding is minimal. First, it is clear that among all semi-modular lattices which contain $\mathfrak{Z}$ as an isometric sublattice, $\subseteq$ has the fewest points, and also the smallest possible number of join irreducible elements. Furthermore, if $\mathcal{Z}$ is already semi-modular, then the normal imbedding lattice $\subseteq$, based on the usual rank function for $\mathcal{R}$, is isomorphic to R. Even if $\mathfrak{R}$ is not semi-modular, $\subseteq$ is isometrically isomorphic to a sublattice of the semi-modular point lattice of Dilworth's imbedding. One might suspect, then, that $\subseteq$ is isomorphic to a sublattice of any semi-modular lattice which contains $\&$ as a sublattice and preserves the rank function originally defined

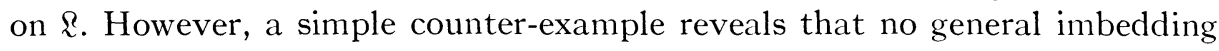
exists which is minimal in this sense. Consider the lattice diagrams shown below, in which the lattice $\mathfrak{R}$, whose elements are denoted by small circles, has been imbedded isometrically in the semi-modular lattices $\subseteq$ and $\Re$, using height on the diagram as rank function. $\subseteq$ is the normal imbedding lattice of this paper, and $\Re$ is clearly the smallest imbedding lattice possible, yet neither is a sublattice of the other.

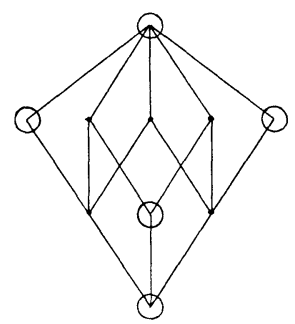

ऽ

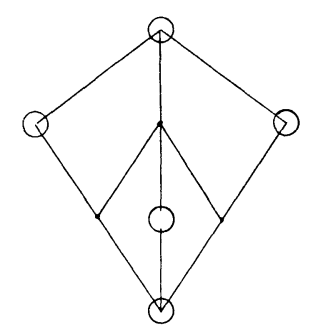

$\mathfrak{R}$ 


\section{REFERENCES}

1. G. Birkhoff, Lattice Theory (rev. ed.), Amer. Math. Soc. Coll. Pub., 25 (1948).

2. R. P. Dilworth, Arithmetic theory of Birkhoff lattices, Duke Math. J., 8 (1941), 286-299.

3. D. T. Finkbeiner, A general dependence relation for lattices, Proc. Amer. Math. Soc., 2 (1951), 756-759.

Kenyon College 